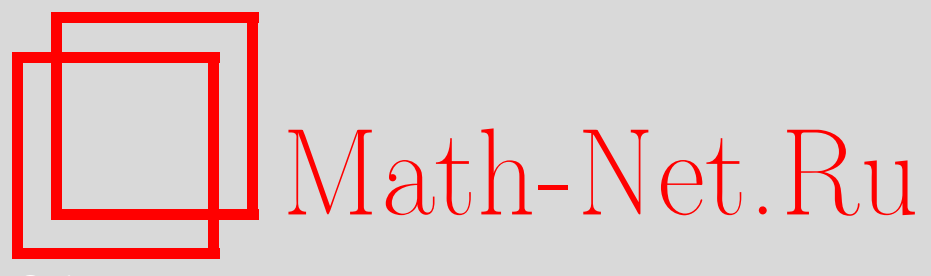

E. В. Лопушанская, Некоторые вопросы аппроксимации обобщенной функции Каратеодори в специальной области $\Omega_{\nu}$, Матем. заметки, 2007, том 81, выпуск 5, 792796

DOI: https://doi.org/10.4213/mzm3726

Использование Общероссийского математического портала Math-Net.Ru подразумевает, что вы прочитали и согласны с пользовательским соглашением http://www.mathnet.ru/rus/agreement

Параметры загрузки:

IP : 54.197 .217 .227

26 апреля 2023 г., 16:50:47

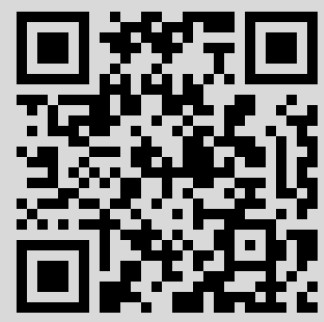




\section{Некоторые вопросы аппроксимации обобщенной функции Каратеодори в специальной области $\Omega_{\nu}$}

\section{Е. В. Лопушанская}

ОПреДЕЛЕниЕ 1. Функция $f$ называется обобщенной функцией Каратеодори, если она мероморфна в открытом единичном круге $\mathbb{D}$ и ядро

$$
K_{f}=\frac{f(\lambda)+\overline{f(\mu)}}{1-\lambda \bar{\mu}}
$$

имеет конечное число отрицательных квадратов.

Обозначим через $C_{\varkappa}$ класс обобщенных функций Каратеодори с $\varkappa$ отрицательными квадратами.

Известно (см., например, $[1 ;$ гл. $\mathrm{V}, \S 3])$, что функция $f$ принадлежит классу $C_{\varkappa}$ тогда и только тогда, когда существует пространство Понтрягина $\Pi_{\varkappa}$, унитарный оператор $V: \Pi_{\varkappa} \rightarrow \Pi_{\varkappa}$ и его порождающий элемент $v \in \Pi_{\varkappa}$ такие, что

$$
f(\lambda)=f(0)+2 \lambda\left[(V-\lambda)^{-1} v, v\right], \quad \lambda \in \mathbb{D} \backslash \sigma_{p}(V) .
$$

Лемма 2. Функиия $f$ удовлетворяет следующим свойствам:

1) $f(\lambda) \in C_{\varkappa}$

2) $\varlimsup_{\substack{\lambda \rightarrow 1 \\ \lambda \in \Omega_{\nu}}} \frac{\operatorname{Re} f(\lambda)}{|1-\lambda|}<\infty$,

3) $\lim _{\substack{\lambda \rightarrow 1 \\ \lambda \in \Omega_{\nu}}} f(\lambda)=0$

тогда и только тогда, когда порождающий элемент $v \in \Pi_{\varkappa}$ в представлении (1) принадлежит области определения оператора $(V-I)^{-1}: v \in \operatorname{dom}(V-I)^{-1}$ и представление (1) функции $f$ принимает вид

$$
f(\lambda)=-2(\lambda-1)\left[(V-\lambda)^{-1} v,(V-I)^{-1} v\right], \quad \lambda \in \Omega_{\nu} \backslash \sigma_{p}(V) .
$$

ДоказАтЕльство. Пусть для функции $f$ выполнены условия 1)-3) леммы. Из представления (1) следует, что

$$
\frac{f(\lambda)+\overline{f(\mu)}}{1-\lambda \bar{\mu}}=2\left[(V-\lambda)^{-1} v,(V-\mu)^{-1} v\right] .
$$

Используя условие 2), имеем

$$
\varlimsup_{\substack{\lambda \rightarrow 1 \\ \lambda \in \Omega_{\nu}}}\left[(V-\lambda)^{-1} v,(V-\lambda)^{-1} v\right]<\infty .
$$

Покажем, что $v \in \operatorname{dom}(V-I)^{-1}$. Пусть $A-\pi$-самосопряженный оператор, связанный с оператором $V$ преобразованием Кэли-Неймана:

$$
A=-i(V+I)(V-I)^{-1}=-i-2 i(V-I)^{-1} .
$$

Работа выполнена при поддержке Российского фонда фундаментальных исследований, грант № 05-01-00203-a.

(C) Е. В. ЛоПУшАНСКАЯ, 2007 
Тогда $\operatorname{dom} A=\operatorname{dom}(V-I)^{-1}$, a

$$
(V-\lambda)^{-1}=\frac{1}{2 i}(\alpha+i)(A+i)(A-\alpha)^{-1}, \quad \text { где } \quad \alpha=-i \frac{1+\lambda}{1-\lambda} .
$$

Из неравенства (3) получаем

$$
\varlimsup_{\substack{\alpha \rightarrow \infty \\ \alpha \in W_{\nu}}}\left[(\alpha+i)(A+i)(A-\alpha)^{-1} v,(\alpha+i)(A+i)(A-\alpha)^{-1} v\right]<\infty .
$$

Так как $A: \Pi_{\varkappa} \rightarrow \Pi_{\varkappa}-$ самосопряженный оператор, то (см. [2; §1]) $\Pi_{\varkappa}$ можно разложить на инвариантные подпространства оператора $A$ :

$$
\Pi_{\varkappa}=\Pi_{\varkappa}^{\prime}[+] \mathrm{H},
$$

где $\Pi_{\varkappa}^{\prime}$ - пространство Понтрягина с $\varkappa$ отрицательными квадратами и $\{\mathrm{H},[\cdot, \cdot]\}$ - гильбертово пространство, причем $A^{\prime}=\left.A\right|_{\Pi_{\varkappa}^{\prime}}$ - ограниченный оператор, а $A^{\prime \prime}=\left.A\right|_{\mathrm{H}}$ - гильбертов самосопряженный оператор. Тогда, разложив относительно (5) порождающий элемент $v=v^{\prime}+v^{\prime \prime}$, перепишем

$$
\begin{aligned}
{[(\alpha+i)} & \left.(A+i)(A-\alpha)^{-1} v,(\alpha+i)(A+i)(A-\alpha)^{-1} v\right] \\
= & {\left[(\alpha+i)\left(A^{\prime}+i\right)\left(A^{\prime}-\alpha\right)^{-1} v^{\prime},(\alpha+i)\left(A^{\prime}+i\right)\left(A^{\prime}-\alpha\right)^{-1} v^{\prime}\right] } \\
& +\left[(\alpha+i)\left(A^{\prime \prime}+i\right)\left(A^{\prime \prime}-\alpha\right)^{-1} v^{\prime \prime},(\alpha+i)\left(A^{\prime \prime}+i\right)\left(A^{\prime \prime}-\alpha\right)^{-1} v^{\prime \prime}\right] .
\end{aligned}
$$

Так как $A^{\prime}$ - ограниченный оператор, то

$$
\lim _{\substack{\alpha \rightarrow \infty \\ \alpha \in W_{\nu}}}\left[(\alpha+i)\left(A^{\prime}+i\right)\left(A^{\prime}-\alpha\right)^{-1} v^{\prime},(\alpha+i)\left(A^{\prime}+i\right)\left(A^{\prime}-\alpha\right)^{-1} v^{\prime}\right]<\infty .
$$

Из неравенства (4) имеем

$$
\varlimsup_{\substack{\alpha \rightarrow \infty \\ \alpha \in W_{\nu}}}\left[(\alpha+i)\left(A^{\prime \prime}+i\right)\left(A^{\prime \prime}-\alpha\right)^{-1} v^{\prime \prime},(\alpha+i)\left(A^{\prime \prime}+i\right)\left(A^{\prime \prime}-\alpha\right)^{-1} v^{\prime \prime}\right]<\infty .
$$

Тогда для $\alpha=i y$

$$
\varlimsup_{y \nearrow_{\infty}}\left[(y+1)\left(A^{\prime \prime}+i\right)\left(A^{\prime \prime}-i y\right)^{-1} v^{\prime \prime},(y+1)\left(A^{\prime \prime}+i\right)\left(A^{\prime \prime}-i y\right)^{-1} v^{\prime \prime}\right]<\infty .
$$

Так как $A^{\prime \prime}=A^{\prime \prime *}$, положив $\sigma(t)=\left[E_{t} v, v\right]$, где $E_{t}$ - спектральная функция оператора $A^{\prime \prime}$, получим

$$
\varlimsup_{y \nearrow \infty} \int_{-\infty}^{\infty}\left|\frac{(t+i)^{2}(y+1)^{2}}{(t-i y)^{2}}\right| d \sigma(t)=\varlimsup_{y \nearrow \infty} \int_{-\infty}^{\infty}\left|\frac{(t+i)^{2}(y+1)^{2}(t+i y)^{2}}{\left(t^{2}+y^{2}\right)^{2}}\right| d \sigma(t)<\infty .
$$

Для вещественной части имеем

$$
\varlimsup_{y \nearrow \infty} \int_{-\infty}^{\infty} \frac{\left(t^{2}-1\right)\left(t^{2}-y^{2}\right)(y+1)^{2}-4 t^{2} y(y+1)^{2}}{\left(t^{2}+y^{2}\right)^{2}} d \sigma(t)<\infty .
$$

Вычислив предел в левой части неравенства, получаем

$$
\int_{-\infty}^{\infty}\left(1-t^{2}\right) d \sigma(t)<\infty \Longrightarrow \int_{-\infty}^{\infty} t^{2} d \sigma(t)<\infty .
$$

Таким образом, по теореме из [3; гл. VI, п. 72] $v^{\prime \prime} \in \operatorname{dom} A^{\prime \prime}$. Следовательно, $v=v^{\prime}+v^{\prime \prime} \in$ $\operatorname{dom} A=\operatorname{dom}(V-I)^{-1}$. 
Покажем, что $\left[(V-\lambda)^{-1} v, v\right] \rightarrow\left[(V-I)^{-1} v, v\right]$ при $\lambda \rightarrow 1, \lambda \in \Omega_{\nu}$. Заметим, что, поскольку $\left((V-I)^{-1}\right)^{*}=-V(V-I)^{-1}$, то $\operatorname{dom}(V-I)^{-1}=\operatorname{dom}\left(V^{*}-I\right)^{-1}$ и

$$
\left[(V-\lambda)^{-1} v, v\right]-\left[(V-I)^{-1} v, v\right]=(\lambda-1)\left[(V-\lambda)^{-1} v,\left(V^{*}-I\right)^{-1} v\right] .
$$

Введем в $\Pi_{\varkappa}$ гильбертово скалярное произведение: $(x, y)=\left(x^{\prime}, y^{\prime}\right)_{1}+\left[x^{\prime \prime}, y^{\prime \prime}\right]$, где $x=$ $x^{\prime}+x^{\prime \prime}, y=y^{\prime}+y^{\prime \prime}-$ представления векторов $x, y$ относительно разложения $(5),\left(x^{\prime}, y^{\prime}\right)_{1}-$ какое-либо каноническое скалярное произведение в $\Pi_{\varkappa}^{\prime}$. Тогда

$$
\left|\left[(V-\lambda)^{-1} v, v\right]-\left[(V-I)^{-1} v, v\right]\right| \leqslant|\lambda-1|\left\|(V-\lambda)^{-1} v\right\|\left\|\left(V^{*}-I\right)^{-1} v\right\| .
$$

Покажем, что норма $\left\|(V-\lambda)^{-1} v\right\|$ ограничена. Операторы $V^{\prime}=\left.V\right|_{\Pi_{\varkappa}^{\prime}}$ и $V^{\prime \prime}=\left.V\right|_{\mathrm{H}}$ связаны с $A^{\prime}$ и $A^{\prime \prime}$ тем же преобразованием Кэли-Неймана. Так как

$$
\begin{aligned}
& \varlimsup_{\substack{\lambda \rightarrow 1 \\
\lambda \in \Omega_{\nu}}}\left[(V-\lambda)^{-1} v,(V-\lambda)^{-1} v\right] \\
& \quad=\left[\left(V^{\prime}-I\right)^{-1} v^{\prime},\left(V^{\prime}-I\right)^{-1} v^{\prime}\right]+\varlimsup_{\substack{\lambda \rightarrow 1 \\
\lambda \in \Omega_{\nu}}}\left[\left(V^{\prime \prime}-\lambda\right)^{-1} v^{\prime \prime},\left(V^{\prime \prime}-\lambda\right)^{-1} v^{\prime \prime}\right]<\infty,
\end{aligned}
$$

то

$$
\varlimsup_{\substack{\lambda \rightarrow 1 \\ \lambda \in \Omega_{\nu}}}\left[\left(V^{\prime \prime}-\lambda\right)^{-1} v^{\prime \prime},\left(V^{\prime \prime}-\lambda\right)^{-1} v^{\prime \prime}\right]<\infty \quad \text { и } \quad \varlimsup_{\substack{\lambda \rightarrow 1 \\ \lambda \in \Omega_{\nu}}}\left\|\left(V^{\prime \prime}-\lambda\right)^{-1} v^{\prime \prime}\right\|<\infty .
$$

Так как $1 \in \rho\left(V^{\prime}\right)$, то

$$
\varlimsup_{\substack{\lambda \rightarrow 1 \\ \lambda \in \Omega_{\nu}}}\left\|\left(V^{\prime}-\lambda\right)^{-1} v^{\prime}\right\|<\infty .
$$

Следовательно,

$$
\varlimsup_{\substack{\lambda \rightarrow 1 \\ \lambda \in \Omega_{\nu}}}\left\|(V-\lambda)^{-1} v\right\|<\infty .
$$

Тогда из неравенства (6) следует, что $\left[(V-\lambda)^{-1} v, v\right] \rightarrow\left[(V-I)^{-1} v, v\right]$ при $\lambda \rightarrow 1$, $\lambda \in \Omega_{\nu}$. Используя равенство (1), из свойства 3) получаем $f(0)=-2\left[(V-I)^{-1} v, v\right]$. Тогда $f(\lambda)=-2(\lambda-1)\left[(V-\lambda)^{-1} v,(V-I)^{-1} v\right]$.

Обратно, легко проверить, что функция $f(\lambda)=-2(\lambda-1)\left[(V-\lambda)^{-1} v,(V-I)^{-1} v\right]$ является функцией Каратеодори,

$$
\varlimsup_{\substack{\lambda \rightarrow 1 \\ \lambda \in \Omega_{\nu}}} \frac{\operatorname{Re} f(\lambda)}{1-|\lambda|}=4 \varlimsup_{\substack{\alpha \rightarrow \infty \\ \alpha \in W_{\nu}}}\left[\frac{1}{2 i}(\alpha+i)(A+i)(A-\alpha)^{-1} v, \frac{1}{2 i}(\alpha+i)(A+i)(A-\alpha)^{-1} v\right] .
$$

Для доказательства свойства 2) достаточно проверить, что

$$
\varlimsup_{\substack{\alpha \rightarrow \infty \\ \alpha \in W_{\nu}}}\left[\frac{1}{2 i}(\alpha+i)\left(A^{\prime \prime}+i\right)\left(A^{\prime \prime}-\alpha\right)^{-1} v^{\prime \prime}, \frac{1}{2 i}(\alpha+i)\left(A^{\prime \prime}+i\right)\left(A^{\prime \prime}-\alpha\right)^{-1} v^{\prime \prime}\right]<\infty,
$$

где $v^{\prime \prime} \in H$ и $A^{\prime \prime}=\left.A\right|_{H}$, а $H$ - гильбертово пространство в разложении пространства $\Pi_{\varkappa}(5)$. Используя, что $\alpha \in W_{\nu}$, т.е. $|t-\alpha| \geqslant|\alpha| \cos \nu$, и то, что $v \in \operatorname{dom} A=$ $\operatorname{dom}(V-I)^{-1}$, получим оценку

$$
\begin{aligned}
\varlimsup_{\substack{\alpha \rightarrow \infty \\
\alpha \in W_{\nu}}}\left[\frac{1}{2 i}(\alpha+i)\left(A^{\prime \prime}+i\right)\left(A^{\prime \prime}-\alpha\right)^{-1} v^{\prime \prime}, \frac{1}{2 i}(\alpha+i)\left(A^{\prime \prime}+i\right)\left(A^{\prime \prime}-\alpha\right)^{-1} v^{\prime \prime}\right] \\
=\varlimsup_{\substack{\alpha \rightarrow \infty \\
\alpha \in W_{\nu}}} \int_{-\infty}^{\infty}\left|\frac{(t+i)^{2}(\alpha+i)^{2}}{(t-\alpha)^{2}}\right| d \sigma(t) \leqslant \frac{1}{\cos ^{2} \nu} \int_{-\infty}^{\infty}\left(t^{2}-1\right) d \sigma(t)<\infty .
\end{aligned}
$$

Свойство 3) следует из представления (2) функции $f$.

Теорема 3. Для функции $f$ следующие свойства: 
1) $f(\lambda) \in C_{\varkappa}$,

2) для челого числа $n \geqslant 0$ существуют $2 n$ вещественных чисел $s_{0}, s_{1}, \ldots, s_{2 n-1}$ такux, чтоо

$$
f(\lambda)+\sum_{\nu=0}^{2 n-1} s_{\nu}(\lambda-1)^{\nu+1}=O\left((\lambda-1)^{2 n+1}\right), \quad \lambda \rightarrow 1, \quad \lambda \in \Omega_{\nu},
$$

выполнены тогда и только тогда, когда существуют $\pi_{\varkappa}$-пространство Понтрягина $\Pi_{\varkappa}$, $\pi$-унитарный оператор $V: \Pi_{\varkappa} \rightarrow \Pi_{\varkappa} u$ порождающий для $V$ элемент $v \in$ $\operatorname{dom}\left((V-I)^{-(n+1)}\right)$ maкue, umo

$$
f(\lambda)=-2(\lambda-1)\left[(V-\lambda)^{-1} v,(V-I)^{-1} v\right], \quad \lambda \in \Omega_{\nu} \backslash \sigma_{p}(V) ;
$$

при этом

$$
s_{\nu}= \begin{cases}2\left[(V-I)^{-(\nu+1)} v,(V-I)^{-1} v\right], & 0 \leqslant \nu \leqslant n, \\ (-1)^{\nu-n} 2\left[(V-I)^{-(n+1)} v, V^{\nu-n}(V-I)^{-\nu+n-1} v\right], & n<\nu \leqslant 2 n-1 .\end{cases}
$$

ДокАзАтЕльство. Представление (7) функции $f$ обеспечивает выполнение условий 2) и 3) леммы, откуда получаем представление (8).

Для доказательства того, что $v \in \operatorname{dom}(V-I)^{-(n+1)}$, введем в рассмотрение функцию $g(\alpha)=i f(\lambda)$, где $\alpha=i(1+\lambda) /(1-\lambda)$. Эта функция является функцией Неванлинны: $g(\alpha)=\left[(A-\alpha)^{-1} u, u\right]$, где $A=i(I+V)(I-V)^{-1}$ - самосопряженный оператор, а $u=$ $(A+i) v$. Из теоремы $[2 ; \S 1,1.10]$ мы знаем, что $u \in \operatorname{dom} A^{n}=\operatorname{dom}(V-I)^{-n}$. Из того, что $(V-I)^{-1} v=-\frac{1}{2 i} u$, следует, что

$$
(V-I)^{-1} v \in \operatorname{dom}(V-I)^{-n} \Longrightarrow v \in \operatorname{dom}(V-I)^{-(n+1)} .
$$

Обратно, заметим, что для $k \geqslant 0$ и $v \in \operatorname{dom}\left((V-I)^{-(k+1)}\right)$ выполнено тождество

$$
\begin{aligned}
& 2(\lambda-1)\left[V(V-I)^{-1}(V-\lambda)^{-1} v, v\right] \\
& \quad=-\sum_{\nu=0}^{2 k} s_{\nu}(\lambda-1)^{\nu+1}+2(-1)^{k+1}(\lambda-1)^{2 k+2}\left[(V-\lambda)^{-1}(V-I)^{-(k+1)} v, V^{k}(V-I)^{-(k+1)} v\right] .
\end{aligned}
$$

Для доказательства представления (7) проверим, что

$$
\begin{aligned}
& -2(\lambda-1)^{2 k+1}(-1)^{k}\left[(V-I)^{-(k+1)} v, V^{k}(V-I)^{k-1} v\right] \\
& \quad+2(-1)^{k+1}(\lambda-1)^{2 k+2}\left[(V-\lambda)^{-1}(V-I)^{-(k+1)} v, V^{k}(V-I)^{-(k+1)} v\right]=O\left((\lambda-1)^{2 k+1}\right) .
\end{aligned}
$$

Для этого достаточно показать, что

$$
(\lambda-1)\left[(V-\lambda)^{-1}(V-I)^{-(k+1)} v, V^{k}(V-I)^{-(k+1)} v\right] \rightarrow 0, \quad \lambda \rightarrow 1, \quad \lambda \in \Omega_{\nu} .
$$

Переходя к $\pi$-самосопряженному оператору $A$, получим, что для доказательства нашего утверждения нужно проверить, что

$$
\left[\left(A^{\prime \prime}-\alpha\right)^{-1}\left(A^{\prime \prime}+i\right)^{k+2} v^{\prime \prime},\left(A^{\prime \prime}+i\right)\left(A^{\prime \prime}-i\right)^{k} v^{\prime \prime}\right] \rightarrow 0, \quad \alpha \rightarrow \infty, \quad \alpha \in W_{\nu},
$$

где $u^{\prime \prime} \in H$ и $A^{\prime \prime}=\left.A\right|_{H}$, а $H$ - гильбертово пространство в разложении (5) пространства $\Pi_{\varkappa}$. 
Рассмотрим следующий ниже интеграл, где $\sigma(t)=\left[E_{t} u, u\right]$, а $E_{t}$ - спектральная функция оператора $A^{\prime \prime}$ :

$$
\begin{aligned}
& \int_{-\infty}^{\infty}\left|\frac{(t+i)^{k+3}(t-i)^{k}}{t-\alpha}\right| d \sigma(t) \\
& \quad=\int_{-\nu}^{\nu}\left|\frac{(t+i)^{k+3}(t-i)^{k}}{t-\alpha}\right| d \sigma(t)+\int_{|t|>\nu}\left|\frac{(t+i)^{k+3}(t-i)^{k}}{t-\alpha}\right| d \sigma(t) .
\end{aligned}
$$

Используя то, что для $\alpha \in W_{\nu}$ имеют место неравенства $|t-\alpha| \geqslant|\alpha| \cos \nu,|t-\alpha| \geqslant|t| \cos \nu$, получаем следующую оценку:

$$
\begin{aligned}
& \left|\int_{-\infty}^{\infty} \frac{(t+i)^{k+3}(t-i)^{k}}{t-\alpha} d \sigma(t)\right| \\
& \quad \leqslant \frac{1}{|\alpha| \cos \nu} \int_{-\nu}^{\nu}\left|\left(t^{2}+1\right)^{k+1}(t+i)\right| d \sigma(t)+\frac{1}{\cos \nu} \int_{|t|>\nu}\left|\left(t^{2}+1\right)^{k+1}\left(1+\frac{i}{t}\right)\right| d \sigma(t) .
\end{aligned}
$$

По теореме [3; гл. VI, п. 72] имеем

$$
\int_{-\infty}^{\infty}\left(t^{2}+1\right)^{k+1} d \sigma(t)<\infty
$$

откуда и получаем нужный результат.

\section{СПИСОК ЦИТИРОВАННОЙ ЛИТЕРАТУРЫ}

[1] Т.Я. Азизов, И. С. Иохвидов, Основы теории линейных операторов в пространствах с индефинитной метрикой, Наука, M., 1986. [2] M. G. Krĕ̌n, H. Langer eng mit der Theorie hermitescher, Math. Nachr., 77 (1977), 187-236. [3] Н. И. Ахиезер, И. М. Глазман, Теория линейных операторов в гилъбертовом пространстве, Наука, М., 1966.

\section{Е. В. Лопушанская}

Воронежский государственный университет

E-mail: kate_lopushanskaya@yahoo.com
Поступило 16.11.2006 